\title{
Sensor-driven agenda for intelligent home care of the elderly
}

\author{
Ângelo Costa ${ }^{\mathrm{a}, *}$, José Carlos Castillo ${ }^{\mathrm{b}}$, Paulo Novais ${ }^{\mathrm{a}}$, Antonio Fernández-Caballero ${ }^{\mathrm{b}}$, Ricardo Simoes ${ }^{\mathrm{c}, \mathrm{d}, \mathrm{e}}$ \\ a Department of Informatics/CCTC, University of Minho, Braga, Portugal \\ ${ }^{\mathrm{b}}$ Universidad de Castilla-La Mancha, Instituto de Investigación en Informática de Albacete, 02071 Albacete, Spain \\ ${ }^{\mathrm{c}}$ Institute of Polymers and Composites IPC/I3N, University of Minho, Guimarães, Portugal \\ d Polytechnic Institute of Cávado and Ave, Barcelos, Portugal \\ ${ }^{\mathrm{e}}$ Life and Health Sciences Research Institute (ICVS), School of Health Sciences, University of Minho, Campus de Gualtar, 4710-057 Braga, Portugal
}

\section{A R T I C L E I N F O}

\section{Keywords:}

Home care

Elderly

Monitoring

Personal agenda

Intelligent agents

\begin{abstract}
A B S T R A C T
The exponential increase of home-bound persons who live alone and are in need of continuous monitoring requires new solutions to current problems. Most of these cases present illnesses such as motor or psychological disabilities that deprive of a normal living. Common events such as forgetfulness or falls are quite common and have to be prevented or dealt with. This paper introduces a platform to guide and assist these persons (mostly elderly people) by providing multisensory monitoring and intelligent assistance. The platform operates at three levels. The lower level, denominated "Data acquisition and processing" performs the usual tasks of a monitoring system, collecting and processing data from the sensors for the purpose of detecting and tracking humans. The aim is to identify their activities in an intermediate level called "activity detection". The upper level, "Scheduling and decision-making", consists of a scheduler which provides warnings, schedules events in an intelligent manner and serves as an interface to the rest of the platform. The idea is to use mobile and static sensors performing constant monitoring of the user and his/her environment, providing a safe environment and an immediate response to severe problems. A case study on elderly fall detection in a nursery home bedroom demonstrates the usefulness of the proposal.
\end{abstract}

(c) 2012 Elsevier Ltd. All rights reserved.

\section{Introduction}

Elderly population growth is a concerning situation. The latest census collected in developed countries show that elderly population is increasing very fast, whilst on the contrary younger population is decreasing. This means that the overall population is getting older, thus triggering scenarios that were unknown in the recent past. The new situation reveals new challenges, mostly related to health, economy and society issues. Medical advances permit the providence of better services to the attendants, as well as the availability of an improved health care to the general public. This translates to better services, procedures and cures to current illnesses. The main beneficiaries of these improvements are persons - most likely elderly persons - whose health condition is more fragile. This population group frequently suffers from illnesses; and severity is more intense than in younger persons (Weil, 2006). Although chronic and constant pain are quite common, new medical approaches to these diseases help people in improving their daily life. The described factors contribute to an increased life expectancy and quality. As aforementioned, the abrupt reverse of the population age pyramid brings adverse economical situations. There is no

\footnotetext{
* Corresponding author. Tel.: +351 253 604435; fax: +351 253604471 .

E-mail address: acosta@di.uminho.pt (Â. Costa).
}

doubt that costs involved in elderly care are elevated. On the other hand, families and public social care are less able to provide services as less money is available to date. Moreover, young working people might support persons in retirement, but currently there is a lack of balance, revealing that about three workers are needed to economically support a single elderly person. The economic problems also lead to social problems (United Nations, 2009). Indeed, health care services, such as daycare centers and retirement houses, are insufficient to accommodate all the needy persons. A more familiar solution is to look after a person at his/her relatives' home. But, this carries an economic and personal burden. The family has to support one more person who normally makes little money as income and much attention has to be spent on the elderly. Such timely or economic sacrifices are usually not assumable by the families (Beard, 2010).

In contrast, elderly persons tend to prefer living at their own homes. But it is difficult to provide the necessary safety and assistance when nobody is monitoring them. Also, as loneliness sets in, the elderly become more reclusive, and the probability of being unassisted when a problem arises increases. In this sense, technology can be the solution to the described problems. The rapid decrease of technological devices' cost and the exponential increase of capabilities and computing power leads to a multipurpose use in new domains. The availability of software that provides 
intelligent and proactive actions, according to sensor data capture, enables the use of complex functions in simple sensors and actuators. Ambient Assisted Living (AAL) is an area that has been intrinsically joined to this concept. AAL describes user-home interactions using sensors and actuators. It also defines the interfaces and devices needed by the user in case of disabilities and illnesses.

\section{Current approaches in intelligent home care}

The concept of Ambient Assisted Living consists in applying technology in a user's home environment, transformed into a seamless interactive sphere adapted to the user's needs (Nehmer, Becker, Karshmer, \& Lamm, 2006). For this reason, the AAL paradigm centers all its developments on the user. It is possible to build systems that modify the environment by using tools such as home automation and informatics systems. The environment is adapted as the information is captured, taking into account the user profile and preferences. A consortium of domotics with intelligent and proactive software applications implements intelligent systems on domestic environments to assist the user and control the environment.

AAL is a very active research area where projects are continuously arising. Currently, the AAL paradigm takes several forms: constituting a hybrid system, according to the devices at the user's home, or attached to the user's body. In this work two commonly used technologies are integrated: cognitive assistants and multisensory monitoring. Both technologies are combined in an interoperable fashion to exchange the information between different concepts. Several processing levels provide a unified framework to assist elderly people in their daily life. Moreover, the development of AAL systems is also supported by methodologies to guarantee success in the transition of the system from a prototype to an industrial product. For example, the work of Botia, Villa, and Palma (2012) illustrates the whole fabrication process for a system capable of detecting falls or similar problems (e.g. heart attacks) of elderly persons who live alone at their own homes.

In order to help the elderly person, computer vision techniques are used at a lower level to track the current status of the user coupled with other kinds of off-the-shelf sensors, impacting in terms of health condition and emergency. The set of sensors and techniques is arranged as a multisensory monitoring component of the system. At a higher level, cognitive assistants keep the user informed and active, by notifying him/her of different events, serving as an intelligent scheduler when the data collected from the lower levels is important. It serves as a gateway between the system and the user, providing him with insightful and simple information about himself/herself.

\subsection{Multisensory monitoring}

Automatic recognition of anomalous human activities in an indoor setting from video sequences is for sure a suitable technology for low-cost home-based health care. In this sense, detection systems based on intelligent computer vision greatly reduce the costs and inconveniences associated with sensor-based systems. These systems usually consist of a series of object detection, tracking and activity interpretation techniques that, combined with a set of heterogeneous sensors (color cameras, infrared cameras, movement sensors, and so on), are able to detect people's current position, movement, as well as the events and activities involved. To date different sensor technologies are proposed to ensure good detection performance whilst keeping privacy in some sensible areas. Current commercial systems, such as range sensors, are less intrusive and a powerful tool to perform low-cost human tracking and activity recognition (Kolling \& Carpin, 2007; Schumacher, Salehi, Hadjiyanni, \& Papanikolopoulos, 2011). In fact, multiple hu- mans can be tracked with this kind of sensors, enabling a comprehensive coverage of the world. The proposal is scalable since multiple sensors can be deployed in the environment. Moreover, the technology enables people to interact with their environment using motion detection algorithms that recognize gestures (Latta et al., 2010). Monitoring is focused on obtaining the current status of the people inside the environment. This status means actions such as standing, sitting or lying (with a very different impact whether on a bed or on the floor). In Bajo, Fraile, Pérez-Lancho, and Corchado (2010), a set of modular services for supervising and monitoring dependent patients living at home is presented. A multi-agent system offers a series of functionalities, including an automatic reasoning and planning mechanism for scheduling the medical staff working day, an alert system, a location and tracking system, and an identification system. Other approaches use spatial-temporal motion representations to capture the relevant velocity information of human actions and combine this information with motion templates to perform the recognition (Olivieri, Gómez Conde, \& Vila Sobrino, 2012). Other works use wireless sensor network technology to detect falls. This is the case of the approach by Paoli et al. (2012) to infer potential falls, where wearable wireless sensor nodes equipped with accelerometers are combined with heterogeneous sensor nodes to obtain a non-intrusive infrastructure.

The previous ideas tackle the topics of environment monitoring and activity detection. Nevertheless, multisensory monitoring is also intended to deal with a set of body sensors to monitor peoples' status in real-time. This enables collecting precise data about parameters such as heartbeat, body temperature, and acceleration speed capture from human-centric wearable or peripheral sensors (see Fig. 1), (DeLong, 2003). This information establishes the health status of the monitored people in real-time, and the system reacts whenever an abnormality is detected at a precise moment. As aforesaid, multisensory monitoring operates at the lower processing levels, being unable to take actions on a detected situation by itself. For this reason, a higher-level module is required to perform decision-making as well as other tasks to act on the environment or to respond with appropriate actions.

\subsection{Cognitive assistants}

Cognitive assistant (CA) is a concept connected to computer and medical science. At its core, the CA connects the two areas in form of software and hardware applications that are specially designed to overcome user disabilities. As AAL is highly centered on the user, a full customization of the platform to each possible user it required. There are several identified cognitive problems (and possibly their combinations) that make user sufferings to require special attention. Memory problems, affecting a wide range of population, are one of the most widespread. Therefore, the most advanced and

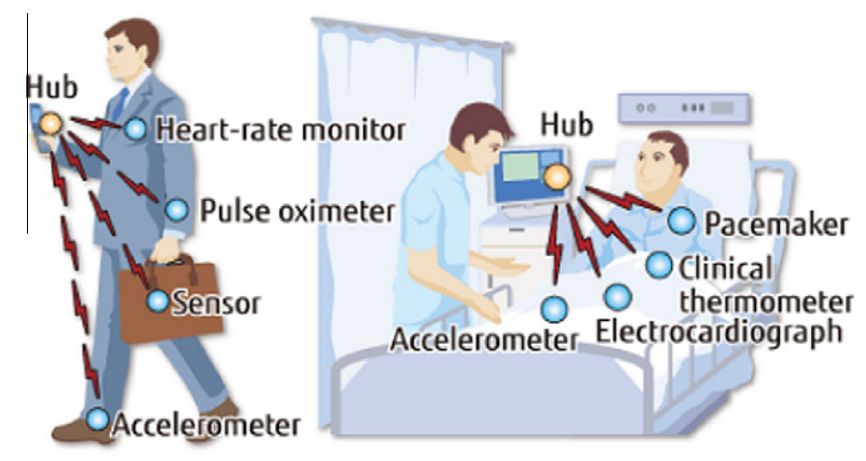

Fig. 1. Example of body sensors deployment. 
acclaimed projects are currently centered on memory problems, mainly due to the number of persons affected. Moreover, there are many elderly persons affected by cognitive disabilities (Jorge, 2001). In fact, after the age of 55 the average person starts to experience cognitive impairment in memory. The cognitive impairment generally progresses linearly, but in some cases it progresses very fast. And, stages such as dementia are reached when measures are not taken (for instance, in form of exercises that excite the memory).

Moreover, cognitive problems severely diminish the capability of executing common daily activities. In case of elderly persons, daily activities are even more difficult as they also are conditioned by mobility. Having episodes of memory lapses makes them disoriented and in danger of hurting themselves in case of a panic attack. Also, disoriented and debilitated elderly persons tend to fall at their homes. If assistance is not provided in time, serious situations use to occur. A lack of user response or an inability to move leads to the impossibility to call emergency services. In an extreme case, the fact of not receiving help in time leads to death. In this sense, cognitive assistants help elderly people by providing useful warnings and by organizing their daily activities. Generally speaking, there are two services to efficiently organize the user's daily activities. The first is scheduling, which keeps the user attentive and aware to his/her programmed activities (even the common ones). The second service is remembering, that is, forcing the user to remember the activities through providing a discrete memory exercise to keep the brain active. An intelligent, fairly and intuitive agenda must be provided to achieve such a result. The agenda has to keep track of the user activities and proactively receive and schedule new ones.

The agenda serves two purposes: (1) scheduling and organizing the user events, and exercising the user memory, providing suggestions for future events; and, (2) proactively organizing and warning the user of important events, such as appointments or taking a medicine. In this sense, a CA does not disorient the user through providing frequent information in a too short spawn of time. With all the structure developed, cognitive assistants also serve for post problems, i.e., when the problems have already occurred. This is the case of falls. When a person falls, not only the detection procedures are essential but also the post detection procedures. This is important, for instance, to verify if the user is well or injured, questioning if help is needed and activating the appropriate response.

\section{Our proposal for elderly intelligent home care}

The processing stack of the platform is the result of two merged concepts, namely, intelligent agenda manager and intelligent monitoring framework. The idea is to use mobile and static sensors performing constant monitoring of the user and his/her environment, providing a safe environment and an immediate response to problems. The platform proposed in this work operates at three different levels. The three levels are arranged through modules each one dealing with different information abstraction, ranging from the raw sensor data to the recognized activities and the decisions made. The lower-level module performs the usual tasks of a monitoring system, collecting data from the sensors and performing its processing to detect and track humans. It is called "Data acquisition and processing". Activities are detected in the intermediate level (under "activity detection"). The upper-level module consists of a scheduler that provides warnings, schedules events intelligently and serves as an interface to the rest of the platform (see Fig. 2). It is denominated "Scheduling and decision-making".

\subsection{Sensors for the elderly home care}

As aforementioned, the use of static and mobile sensors is proposed for monitoring the elderly at home. Concerning static sensors, video cameras are used due to our previous long experience in these kinds of sensor devices. Let us remind that analyzing a video scene entails two large phases. On the one hand, there is the usual first phase in object detection, namely segmentation (e.g. Castillo, Serrano-Cuerda, \& Fernández-Caballero, 2010) and tracking (e.g. Fernández-Caballero, Gómez, \& López-López, 2008). This phase consists of capturing images, analyzing them for shape interpretation and, afterwards, recognizing them throughout the scene. On the other hand, there is the higher-level processing, that is, the scene interpretation (context recognition), meaning the analysis of the scene at a global scale. The monitoring capabilities involve several aspects such as vital signs, movement, objects placement, temperature, and appliances, among others. This is, there are different sensing systems collecting different data and standards that have to be considered.

For this purpose, mobile sensors have to be inserted in the platform. In fact, a mobile platform is an important gateway to the rest of the desktop platform. Paired with the desktop version, the mobile application enhances the following aspects: availability and data capture. The availability concept presented here means that the user is connected anywhere and everywhere with a mobile device. Indeed, accessible interfaces are constructed to provide important information with the minimum learning process and without requiring direct user interaction to obtain information. Ughetti, Trucco, and Gotta (2008), a free and fast evolving operating system present in a large range of mobile devices, is adopted to support the mobile platform. The mobile platform is optimally developed to interact with the users, providing visual and audible information and warnings. Also, the mobile platform enables availability to the user everywhere. This means that it is easier to the user to provide feedback when required, without having to even move from where he/she is. The type of interaction that is intended to be performed with the user is very informal, consisting of questions and relevant information about the current status of the system.

Furthermore, mobile device behaves as a port for further sensor capture, since most mobile devices include a large availability of sensors. Sensors such as accelerometers, global positioning systems (GPS), g-force, luminosity, among others, combined with the connectivity inherent to these devices, create a great platform to capture data and transmit it to other platforms. The device is intended to be carried by the user at all times, being able to capture all the data from the available sensors in form of a body area network (BAN). This network works with cheap sensors already present in the device. These sensors support the rest of the platform by providing useful information about their readouts. For instance, an accelerometer and a g-force possibly determine if a person has fallen or had an impact, the GPS determines the person's position, and luminosity determines the proximity, among other interpretations. The collected data is directly used or saved for knowledge extraction, relating the information present in the platform with the occurrences in the mobile device. It is possible to determine which sensor is the best to help in an event such as a fall with this merged information. The data stream is used in the future to create an early warning system. This means that several different systems provide fused information that is used to construct a very accurate sensing platform.

Also, the mobile platform serves an emergency situation. If the user has fallen or is inactive for a period of time, the platform is prompted to present a question to the user, collecting different aspects beyond the answer itself. The lack of response from the user points out that something has happened to the user. Also it can serve as a panic button that makes a call to a relative or an emergency service, automatically providing the user's location. Related projects in portable monitoring have also developed hardware and software that is coupled with complex mobile devices such as 


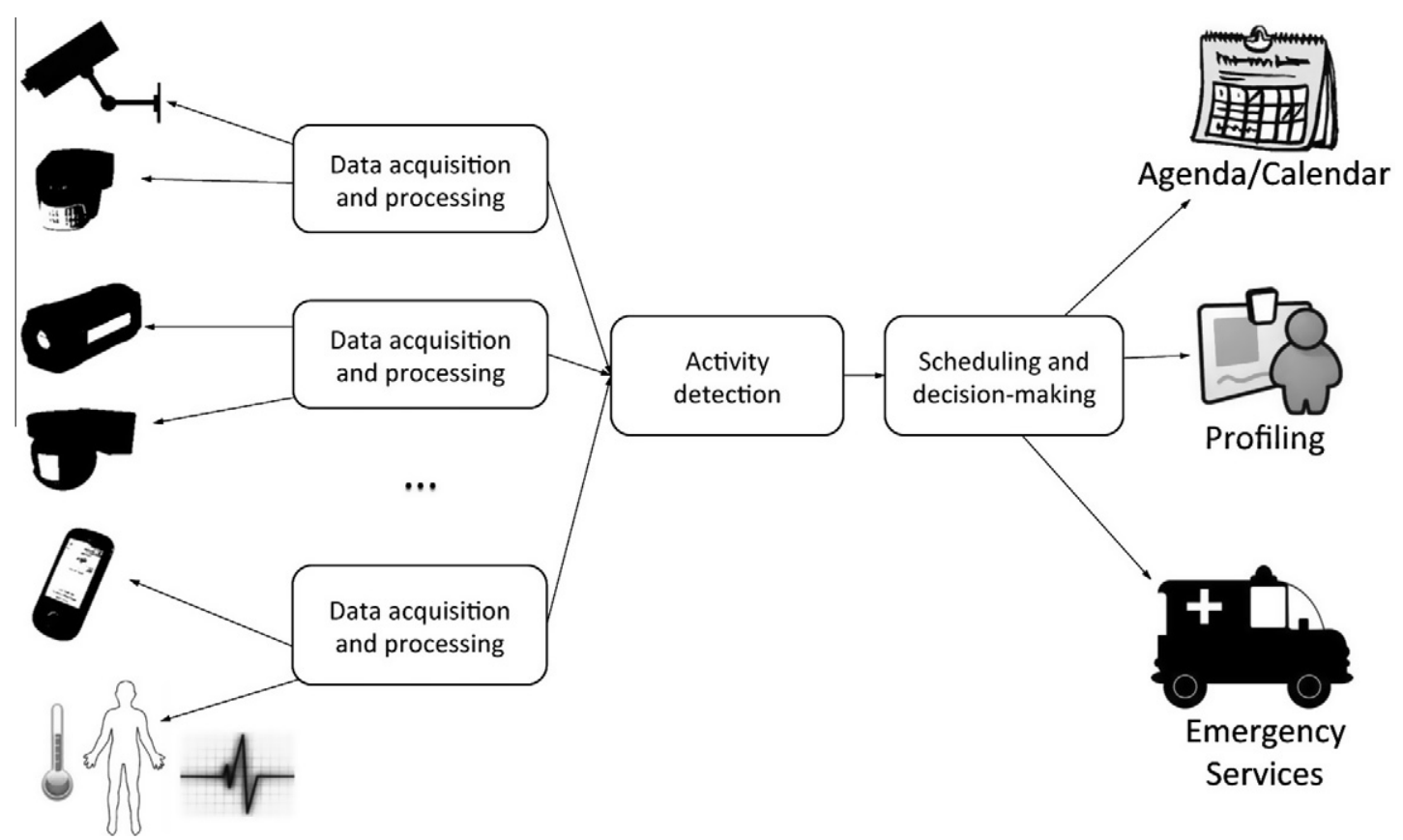

Fig. 2. Platform modules and their components. Sensors and services involved.

smart-phones. This enables to have an extensive array of sensors, thus creating a sensor network (Costa, Barbosa, Melo, \& Novais, 2011). This sensor network is applied to advanced forms of medical mobile monitoring, collecting data such as electrocardiogram (ECG), blood pressure, oximetry, and general vital signs. In this sense, the BAN is fully capable of having pre-emergency detection to feed an inference engine capable of making decisions. The decisions vary from logging the general health condition to warning medical services of a possible life-threatening event. Indeed, heart attacks are detected by sensors even before a person notices it (Leijdekkers \& Gay, 2008).

To sum up, the mobile platform is an important part of the system, being a bridge of the user interaction with the rest of the platform and a monitoring tool by itself. It serves with unnoticed tools such as calendar and positioning system, which provide extended services without any hassle to the user.

\subsection{Services for the elderly home care}

Following the base concept of the iGenda project (Costa, Novais, Corchado, \& Neves, 2011; Costa, Castillo, Novais, Fernández-Caballero, \& López, 2012), the use of different AmI-based monitoring implies that the system becomes more complete and accurate, thus providing a better service to the user. The platform is designed mainly to assist the elderly user, so the problems that most occur with them should be the ones that have more focus. In this sense, a fall detection system is an interesting complement to the iGenda system. Indeed, a fall is a problem that increases in the elderly age with a high rate of occurrence, causing health problems in the long term. Also, falls use to indicate bigger problems, such as loss of cognitive capabilities or underlying cerebral deceases. Of course, an enhanced use is gotten through combining the fall detection system and an early alarm system. A logging system with capability of taking intelligent decisions is provided to immediately call the emergency services or to analyze the overall status before taking proactive measures. An example of such a measure is to schedule an appointment with a doctor or a social assistant. An aggregated service is to create an array of components that provide services such as intelligent scheduling and active monitoring.
The proposal also introduces the context of the ambience surrounding the user. This way, user events that are uncontrolled are sensed, and actions regarding the sensed context are fired to prevent further problems and act over the present ones.

Next, the complete architecture of the platform is presented, showing the components, connections and functions.

\section{Description of the platform}

This section defines each one of the proposed platform modules, emphasizing their main functionalities. A detailed view of the processing components involving the three platform modules is described (see Fig. 3).

\subsection{Execution model}

The execution model that manages each component's functionality is described prior to detailing the main modules of the platform. The proposed platform is defined as a hybrid (or hierarchical) distributed system, where a series of remote nodes perform part of the processing (the lowest levels), whilst other higher level nodes collect the results of the low-level processing to obtain higher level information (see Fig. 3). This implies the existence of a series of processing components where connected sensors communicate with other high-level modules. The main difference with other execution models, such as distributed models, is that not all components perform the same operation. The arrangement is hierarchical, the high-level modules being in charge of controlling the low-level ones. The distribution of the system operation is necessary, given the requirements inherent to a system in charge of performing monitoring tasks from several data sources, as well as of detecting activities, performing the user profiling and intelligent planning of the user agenda. In short, the proposed platform has high computation requirements with a need for real-time response.

The selected execution model is commonly used in state-of-theart monitoring systems. Some examples are found in the work of Castro, Delgado, Medina, and Ruiz-Lozano (2011), where an 


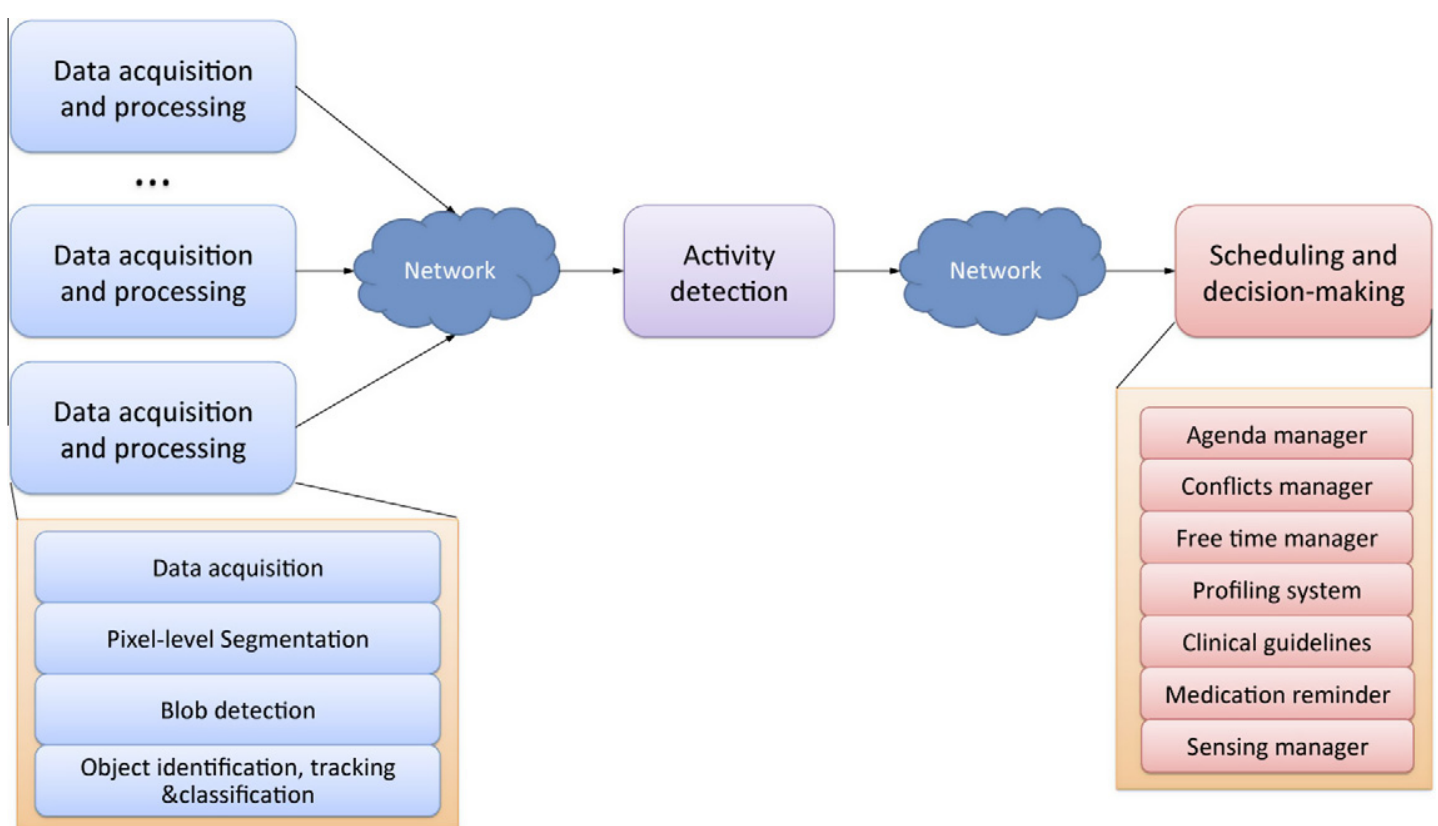

Fig. 3. Main components of the platform modules.

intruder detection system, which integrates heterogeneous information sources, is presented. There are other works, as in Vallejo, Albusac, Gonzalez-Morcillo, and Jimenez (2008), where a serviceoriented multi-agent surveillance system is proposed. The proposal consists of a two-layer organization where some agents are distributed through the medium to capture information from sensors whilst a central node gathers and processes the information.

\subsection{Data acquisition and processing}

Each low module of the platform is in charge of interacting with the analog devices (sensors) measuring from the real world. As the sensors are scattered in the continuously measured environment, it is important to provide processing mechanisms able to adapt to the requirements. For this reason, the components are arranged in a decentralized manner. The modules' operation is considered lineal in the sense that it is composed of a set of operation components, each one in charge of a specific task. The proposed components are complementary, the execution of one component to start the next one being necessary. It is possible that several algorithms operate at the same component level, but some synchronization mechanisms are needed to organize their execution. Seven components are associated with these modules, corresponding to the traditional stack of image processing. The association with the image processing stack is due to the fact that these sensors are the ones that provide more information. As shown in Fig. 3, a "Data acquisition and processing" module includes the following components:

- Data acquisition

- Pixel-level segmentation

- Blob detection

- Object identification, tracking \& classification

The Data acquisition component translates data from the sensors. It is usual that sensor information contains noise. The component is in charge of solving this issue by a series of preprocessing techniques, adapted to each specific sensor. For example, in image processing, apart from the noise filtering, it is quite common to apply filters to enhance color or contrast. Given the preprocessed input information, the next step is to isolate candidate spots corresponding to the objects of interest. Thus, the Pixel-level segmentation component consists of a wide range of algorithms devoted to human segmentation, ranging from simple techniques such as image or background subtraction to other more complex ones like motion templates (Castillo et al., 2010; Fernández-Caballero, Castillo, Martínez-Cantos, \& Martínez-Tomás, 2010).

Following with the low-level processing stack, the Blob detection component is in charge of filtering the spots candidates to objects of interest to eliminate misdetections. This filtering enhances the detected spots' contours, grouping spots incorrectly split during the segmentation process as well. The current component also improves the information abstraction, passing from image information (or combined to sensor information) to blob information, extracting high-level information from the spots to allow a more efficient object identification in the next levels.

The next component is in charge of the Object identification, tracking \& classification to raise the information abstraction from blobs to objects (possibly composed of several blobs). To distinguish whether one or several blobs belong to one object, their coordinates in the image space are translated to real world coordinates. Besides, whilst blob information is considered instantaneous (blobs appearing in consecutive frames are unrelated), objects remain along time. Moreover, information regarding objects exists beyond the sensor coverage range (the object properties remain associated to each object, even though the object is detected by a different sensor). Regarding object classification, this component also provides information about "what" the objects are, which is essential to perform an accurate event and activity detection. Finally, to complete the objects' analysis, it is necessary to include tracking capabilities to the platform. In this sense, the system calculates the trajectories followed by the objects in the scenario as well as to predict their future positions.

\subsection{Activity detection}

Many techniques and methods have been used so far in human activity recognition and understanding. According to Huang, Wang, 
Tan, and Maybank (2009), shape features and spatial-temporal features are often used for single person activity analysis, and motion features are used for interactive person activity. Besides, two main component of the event understanding process - abstraction and event modeling - are key aspects in the activity detection process. Abstraction corresponds to the process of molding the data into informative units to be used as input to the event model (Natarajan \& Nevatia, 2008), whilst event modeling is devoted to formally describing events of interest and enabling recognition of these events as they occur in the video sequence (Ulusoy \& Bishop, 2005).

The proposed algorithm incorporates external knowledge about the problem domain into the expected structure of the "Activity detection" module. Motion-based image features are explicitly linked to a symbolic notion of hierarchical activity through several layers of more abstract activity descriptions (Fernández-Caballero, Castillo, \& Rodríguez-Sánchez, 2010). Atomic actions are detected at a low level and fed to handcrafted grammars to detect activity patterns of interest. Besides, the algorithm works with shape and trajectory to indicate the events related to moving objects. Interpreting a visual scene is a task that, in general, resorts to a large body of prior knowledge and experience of the viewer (Neumann \& Möller, 2008). Through the actions and events described, basic patterns (an object speed or direction) and more complex patterns (e.g. a fall) are found out. It is essential to define the desired behavior pattern for each situation. Thus, for each specific scene a state diagram and a set of rules are designed to indicate the patterns. In comparison to other approaches, such as Bayesian networks or hidden Markov models (Oliver \& Horvitz, 2005), ours is unable to model uncertainty in video events. But it is presented as a useful tool in video event understanding because of its simplicity, its ability to model temporal sequences and easily incorporate new actions.

\subsection{Scheduling and decision-making}

The scheduling interface with the user is developed following the principles established in the iGenda project. It constitutes the higher-level data processing, receiving data from the other modules through direct communication or by using communication protocols such as SOAP (Englander, 2002). Intelligent scheduling goes beyond the normal conception of traditional scheduling. The intelligent agenda contains several events in charge of actions such as taking medication, which persons normally carry out but are difficult to remember for a memory impaired person.

Cognitive assistants are more than applications that simply help persons to remember. They are memory stimulants and are able to connect a person with a complex system in an intuitive way, providing simple and informative responses from events generated by the platform. In this sense, the platform goes beyond the CA concept. The proposed system has the objective of providing not only a cognitive assistant in form of an intelligent scheduler, but also a centralized platform that connects users, relatives, doctors, and computer agents. In terms of scheduling, the platform processes two different aspects: direct scheduling and playful activities management. As a playful activities manager, it schedules activities in the free slots of the calendar taking into account the user profile. So, the events are chosen minding the user conditionings, diseases or mobility conditionings, as well as the weather and environmental variables. The direct scheduling forces the processes needed to schedule an event appointed by the user or another person. It relies on decision engines to verify if there are conflicts and solves them in the best possible way, verifying several conditions inherited by the events and sorting the events. The process is based on production models of scheduling (Pacciarelli, Meloni, \& Pranzo, 2011).

The scheduling of playful activities are intended to keep the person occupied and active. This is done by forcing the person to think about the activity in question, and exercising the baring by remembering and memorizing certain aspects of the activities. Following these ideas, activities that require some mental processing are fully recommended to keep the brain active. In fact, some general medical community studies indicate that the best way to prevent further memory loss is to perform activities that require some mental power. Games designed to heavily interact with the user and which require responses from the user to progress in the game are some of the recommended activities. Concentration, memory and math games are very stimulant and demanding, being perfect examples to present to a cognitive impaired person. The overall goal is to keep the user active by suggesting activities that stimulate the mind.

The scheduling algorithm relies on comparison methods to achieve a successful execution. The scheduling is naturally divided into two major parts: conflicted and non-conflicted. They are the direct opposite, conflicted being the activities that clash with others, while the the events that have a free time space in the agenda are non-conflicted. When there is no conflict between activities, the incoming activity is directly saved. Problems emerge when there are conflicts between scheduled and incoming events. The method of resolution follows a hierarchic configuration. The events possess associated features that define their importance value. A two-step validation of the importance value is defined to prevent deceiving the system by tagging values that are not true in the attempt to force an event. The events have two importance values: internal and user profile. The internal value is attributed by the person who schedules the event. This value is normally informative of the urgency of the event, representing a relative importance in the calculation of the importance of the event. The platform considers user profiles for each user. The module balances the values and provides a weighted value. At this moment it is an $80 \%$ to $20 \%$, in favor of the profile value. In Table 1 an example of the values association is shown. The events are then compared and a result is achieved among the following possibilities (see Fig. 4):

- Remain: The scheduled event is more important than the newer, so there is no exchange. The sender and receiver users are notified.

- Change: The new event replaces the previous one, notifying the sender and, if configured, notifying the receiver. The old event is eliminated and other users participating in that event are warned.

- Reallocate: This is the most common option. It enables the reallocation of the current (or new) event to new available empty spaces. Either the current event is reallocated to a new position, permitting the new event to be scheduled, or the new event is reallocated in another vacancy time slot. The operation is automatic, semi-automatic or manual, defining the way in which the users are notified and the level of control they have over the operation. The warnings are configured to adjust to the user, minding his/her profile.

- Ask: The ask feature is the most user-dependent. This feature relies on some of the previous operations, but only if the values of the events are completely different. Moreover, the user is

Table 1

Importance values from different users; lower is better.

\begin{tabular}{lccc}
\hline User & Internal & User profile & Final \\
\hline Doctor & 0 & 0 & 0 \\
& 10 & 0 & 1 \\
Friends & 0 & 10 & 10 \\
& 10 & 23 & 38 \\
\hline
\end{tabular}




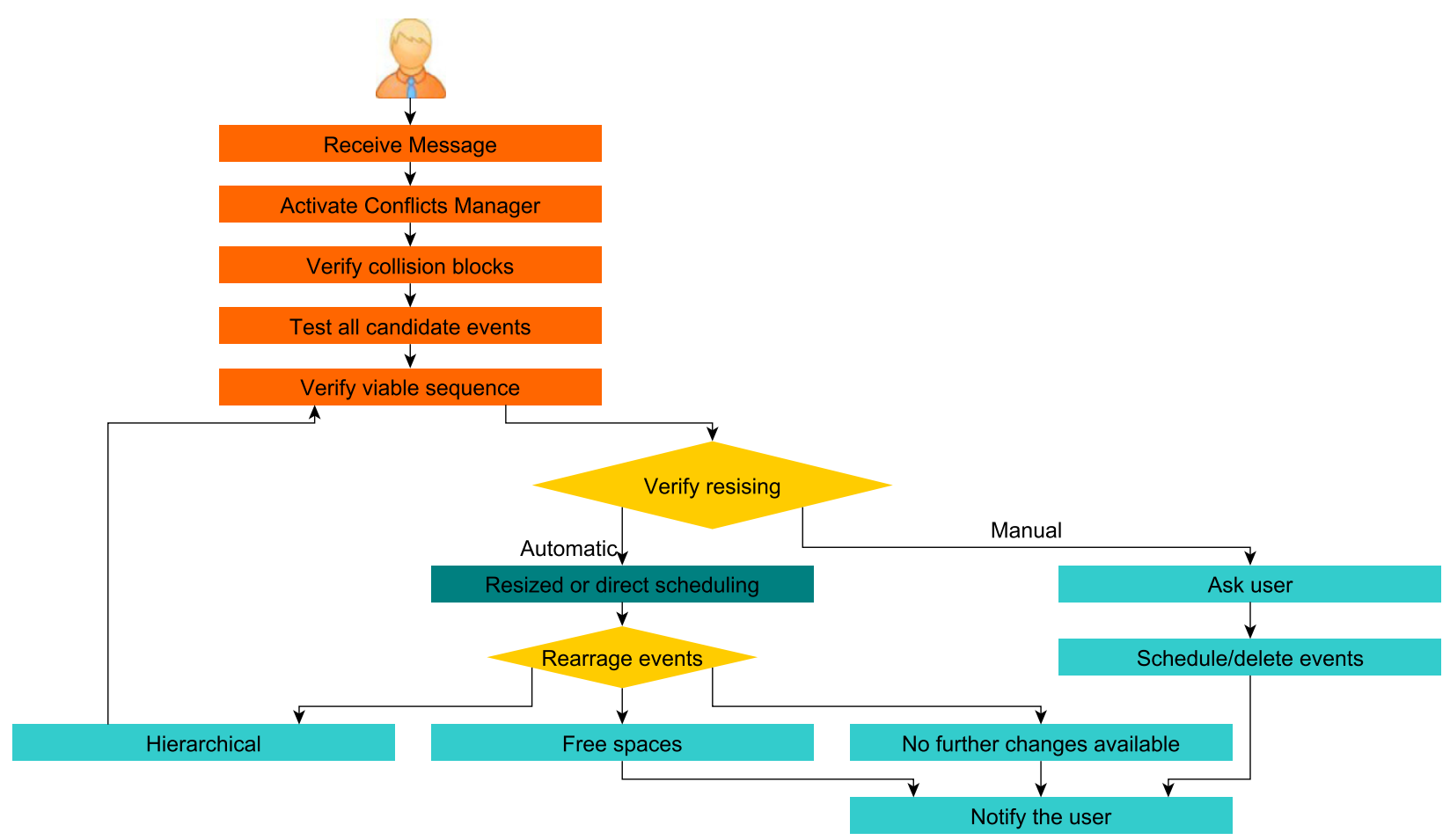

Fig. 4. Schedule and decision-making event handling.

warned if there is any change of the events or a new scheduling, asking if he/she accepts it or not. This means that the user has full control over it.

The module is designed following the multi-agent paradigm, where a set of agents cooperate to perform some specific tasks, being each agent responsible of a functionality. The proposed agents are heavily based in logic functions, which are inspired in production and scheduling management systems. The logic functions assure the correctness of the decisions provided, thus only the profile and the configuration systems being the responsible characteristics that change the variables and methods of operation. The agent platform provides the tools needed to develop a robust and secure system. There is an agent solely dedicated to secure the user information of attacks, filtering the content of the incoming messages. Also, the platform is in charge of launching specific agents for specific tasks, relieving the system overload of having multiple agents enabled but inactive. The platform also detects if an agent is responsive. Therefore, it resets an agent if needed. This means that it is easier to update and to repair certain agents' characteristics and functions without deactivating all the platform. Thus the availability of the platform is constant. As the agents perform different tasks and are complete and robust, they are considered as the components of the "Scheduling and decision-making" module.

The module is constituted by seven agent-based components: (1) The agenda manager controls all incoming communication and launches the agents which deliver the information to the correct agent. (2) The Conflicts manager controls the incoming events, sorts and schedules them. The agent is able to move or delete events in accordance with user preferences. (3) The Free time manager controls the scheduling of leisure activities that mentally and physically stimulate the user. This agent uses the profile information to choose the best activity for the user. (4) The profiling system uses case-based reasoning (CBR) and Bayesian networks (BN) to build a dynamic user profile. It collects and improves the user profile, adjusting it to the user's current likes and conditionings. (5) The Clinical guidelines agent consists of computer interpretable guidelines that provide services to help in triage and diagnostic of health problems. It uses the classical concept of medical guidelines, providing a flowchart-like interface and an interactive environment to the user. (6) The Medication reminder controls medication, keeping in mind the short time frame involving taking medication that might overwhelm the calendar with events. Moreover, these events have different warnings and communication interfaces towards the user, creating also a direct channel with doctors and health care services. So, the medication is updated in real-time (e.g. the dose according to certain parameters). (7) The sensing manager provides proactive measures when an emergency is detected. This agent is an implementation of a body area network (BAN), that is, a portable sensor network with discrete sensors reading vital signs.

\subsection{Data gathering}

There are two different methods in charge of collecting the data concerning the activities, as well as of selecting how the activities are scheduled. Firstly, there is the direct reception of the events done by the agents in the module. Typically, a message uses an XML format in the content and follows the FIPA-ACL protocol (Foundation for Physical Agents, 2002) message structure (see Listing 1). The messages follow high standardized ontologies to proceed with complex communications. These communications transmit internal content such as command codes and error reports. As the users schedule a new event, the user personal platform checks with the receivers trough a notification. Then it starts the internal processing, sorting and scheduling, according to the rules defined. This provides useful information on the event and notifies about the final result of the process.

The second method manages the user's pattern and profiles. The user sustains an intrinsic and complex profile, including what his/ her likes and dislikes are. The user profile drives the actions and 


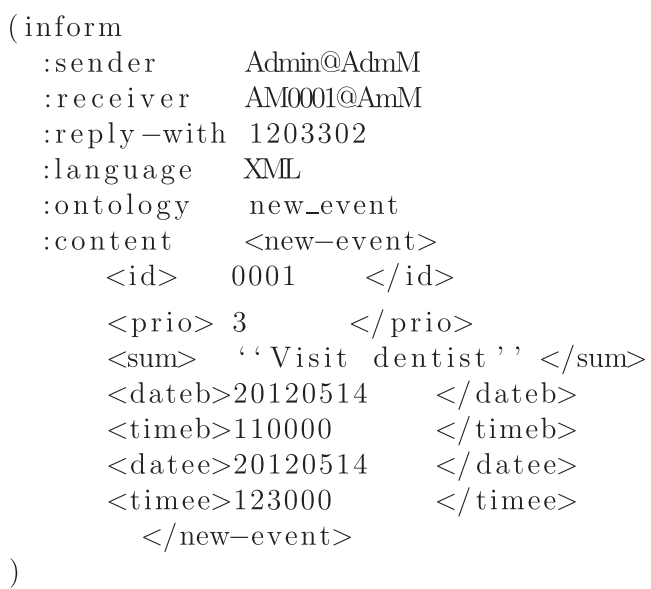

Listing 1. Internal platform messages.

the activities he/she chooses to execute. Detailed information, in the form of a personalized and attentive service, is collected about the monitored person and changes in the environment to provide a useful service in accordance with the profile of the user. The system is able to better accommodate the user in a non-static and non-standard way with this information. This means that the user is benefiting from intelligent services that attend not only to specific illnesses and limitations, but also to the preferences in terms of activities. Besides, the profile contains the importance of the persons that interact with the user. The ranking is a soft form of influencing the method of scheduling new events. The rank of the persons is not determined to be used in a restrictive and discriminatory sense, but to provide better knowledge about the contacting person. For instance, the user's personal doctor should be of the uttermost importance, and the family seconding and so forth. The ranking is constructed based on the user preferences too (e.g. if the user prefers friends or family first, the system updates his/her preferences).

In terms of the "Free time manager" (FTM) agent, the events scheduled or suggested are heavily based on the user preferences. Having the information of what the user needs most and what he/ she likes, the process uses certain types of ranks to calibrate and to provide suggestions accordingly. Therefore, backing the suggestion system, there is a profile system that serves as a preemptive method of sorting and availing the incoming events by verifying the person and internal classification and providing the correct value to the user. The corrected value indicates how much appropriated the event is to the user, adjusting the priority to the expected events of his/her daily life.

All the data collected are saved for future learning. The learning process consists in an agent sub-platform that operates following the CBR and BN concepts. Merging these concepts provides an optimized result by gathering all the available information, user preferences and external variables, and providing the optimal event to be executed (Marques, Costa, \& Novais, 2011). The user preferences are saved in the BN and CBR fields, serving as the field that influences the others by adding $10 \%$ more to the current value, if the event is accepted to be executed by the user. The external variables consist in different information that can be included in the platform such as weather conditions, current localization, or health conditionings. These external variables have the rest of the weight. For instance, if there are two suggested events, as for instance "walk outside" and "read a book", conditions such as being a sunny or rainy day directly influence the chosen activity if they have a close preference value (see Fig. 5).

The CBR modifies the system values by collecting all the events and choices of the user and saving them into a database. The database is then processed in terms of knowledge extraction to identify certain unnoticed aspects and relations. Thereon, the results are aggregated to the present events, modulating the characteristics and the combined relations that the events have with each others. This means that the next suggestions are closer to what the user is expecting, providing a seamless experience with the platform, interacting as less as possible with it. Also, it means the achievement of a dynamic profiling system that corresponds well with the user real preferences. Persons do not have always the same preference towards an event, changing preferences in terms of "most liked", showing a preference to different things at different times. The platform is then able to provide the changes required by taking into account the user responses to the suggested events and immediately modifying the importance of these events.

The data from the BN and CBR is stored in the "Events database". The database is responsible to archive the events and linked characteristics. The database provides the linking between the complete platform to provide further internal tuning and availability. Also, the "Users database" is intrinsically connected to it, providing information about which users are performing the same activity and which activities are mandatory (as shown in Fig. 6).

\section{A case study on elderly fall detection}

For the sake of a better understanding of the proposed platform, a case of study is presented next. It explains the system technological establishment and the interaction and decisions taken after the events occur. The case study presents a very common potentially

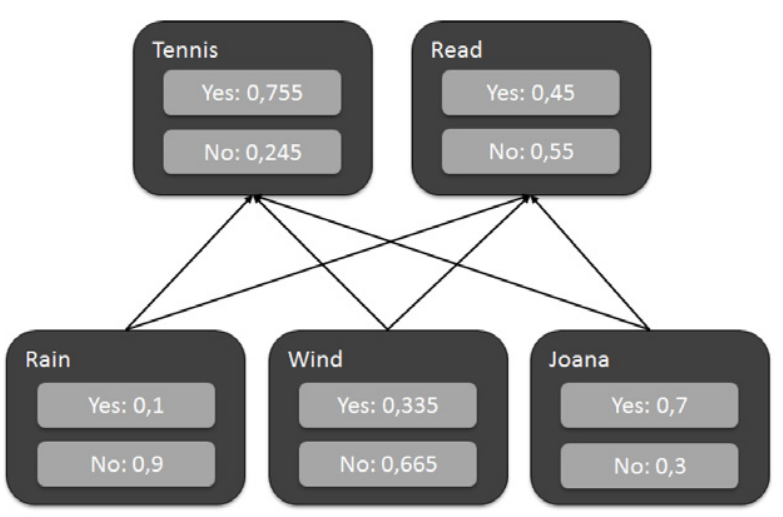

(a) Initial values of the characteristics.

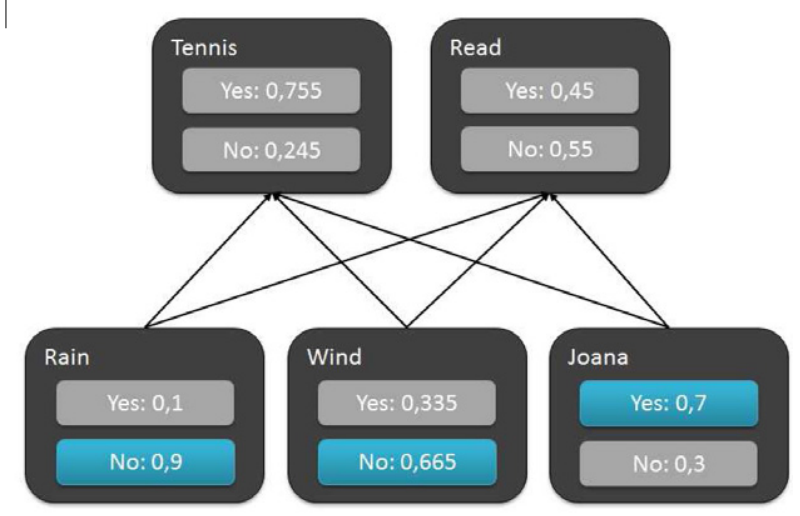

(b) Final results after selecting the appropriate conditionings.

Fig. 5. Bayesian network with external conditions and user preferences 


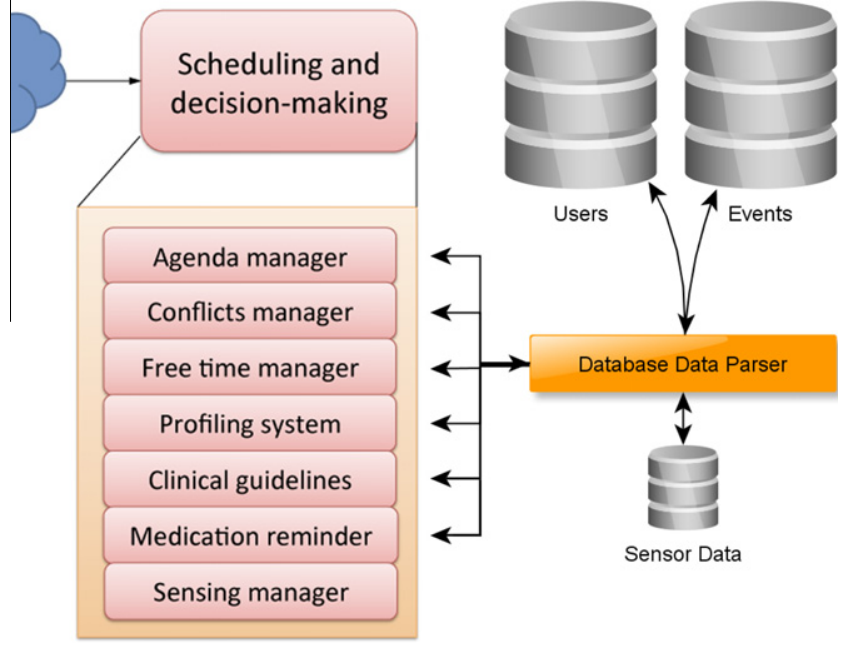

Fig. 6. Expanded view of the platform modules.

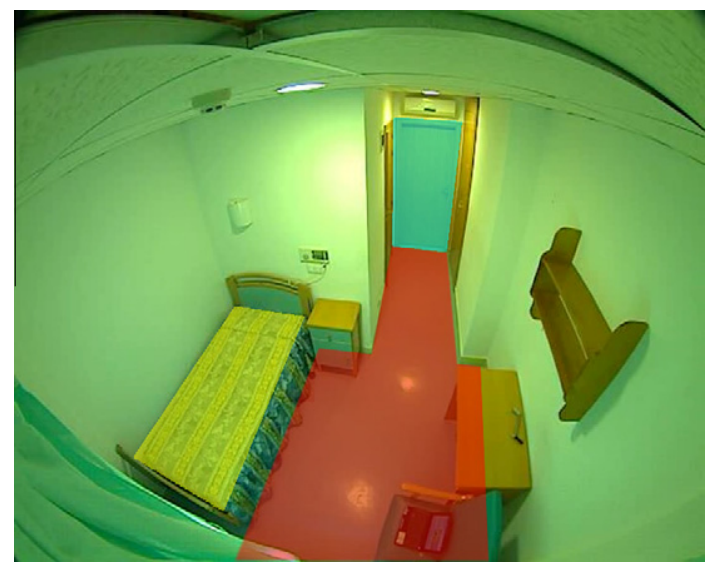

Fig. 7. Test environment. A nursing home bedroom.

dangerous situation which occurs in nursing centers, namely a fall. For this purpose, a real environment where an actor simulates a fall event is presented. Fig. 7 introduces the scenario where three main areas are highlighted. In first place, the entrance door is distinguished in blue ${ }^{1}$. The entrance is the point where human detection is started. The next area considered is shown in yellow and corresponds to the bed surface. This area is considered as people lying on their bed is a very different situation than people lying on the floor. A third area is drawn in red and represents the focus of the fall analysis. As aforesaid, if a human is detected lying on the bed, this is considered as a normal situation, whilst a human lying on the floor generally corresponds to a fall. Images of the case study are taken from a top view provided by an Axis 212 PTZ wide-angle camera installed in a nursing home bedroom.

As the case study involves just one room and one visual sensor, the number of platform modules is reduced. As shown in Fig. 8, only one module is necessary for the low-level processing ("Data acquisition and processing") of the camera information. Regarding "activity detection" and "Scheduling and decision-making", both modules are included to perform a full analysis of the fall event. The next sections correspond to the numbers in the figure and describe the outcome expected for each module.

\footnotetext{
1 For interpretation of color in Fig. 7, the reader is referred to the web version of this article.
}

\subsection{Data acquisition and processing - Human detection}

Human detection is divided into four phases. Firstly, data acquisition is continuously acquiring images from the camera (see the first row of Fig. 9). Humans are segmented to distinguish them from the rest of the scene. The information is used to classify the objects, considering just humans for the activity analysis. The tracking phase enhances the fall detection as it allows to assign a unique identifier to the same object (human) along time, making it possible to keep a history record associated to the humans and to predict their future positions.

\subsection{Activity detection - Fall detection}

The system operation continues with the fall detection module in charge of the analysis of behaviors in the monitored scene. As shown in Fig. 9 five time instants are considered to compose a fall sequence. As aforesaid, a human lying on his/her bed is quite similar (in terms of shape recognition) to a human lying on the floor. For this reason, both areas are differentiated. Table 2 shows the results of the "activity detection" corresponding to the five time instants. It is shown that time rapidly decreases among detected activities given the nature of a fall (from frame 468 to frame 487). Also, although in frame 481 the falling action is initiated, the system detects a crouching event in this case. This consideration is taken to avoid errors, as it is possible that the fall finally does not happen if the person reacts adequately.

\subsection{Scheduling and decision-making}

In terms of "Scheduling and decision-making", as stated before, the platform interacts with the user to determine if there is really an emergency or if just a minor accident or false fall has occurred. This is in fact a double edged sword; no detection should pass ignored, but spending useful resources in non emergencies is also bad due to the limited available resources. Minding these facts, the system is built with several features that enable intelligent decision making and user interaction. This means that the user relies on automatic actions and he/she is also able to interact when the results/decisions are not accurate enough. The following three components play a crucial role in this case:

- Clinical guidelines

- Profiling system

- Agenda manager

These actions work by changing the way the user perceives the events and how he/she responds to them. This modulates the outcome to the best scenario possible. The availability of a mobile platform is important because it supports the interface that enables the user to interact with the platform. For instance, if a question/answer is needed, in case of a fall, the least movements possible and the immediate availability of a device that the user can call for help is the best tool we can provide to the user.

\subsubsection{Clinical guidelines - Warning mechanism}

A warning mechanism is built within the Clinical guidelines component to provide an array of different services that handle warnings, being also a communication interface with the user. The warning can be oriented to emergency services or relatives, or present itself as a method of communicating with the user. As shown in Fig. 10, the procedure after detecting a fall is as follows: 


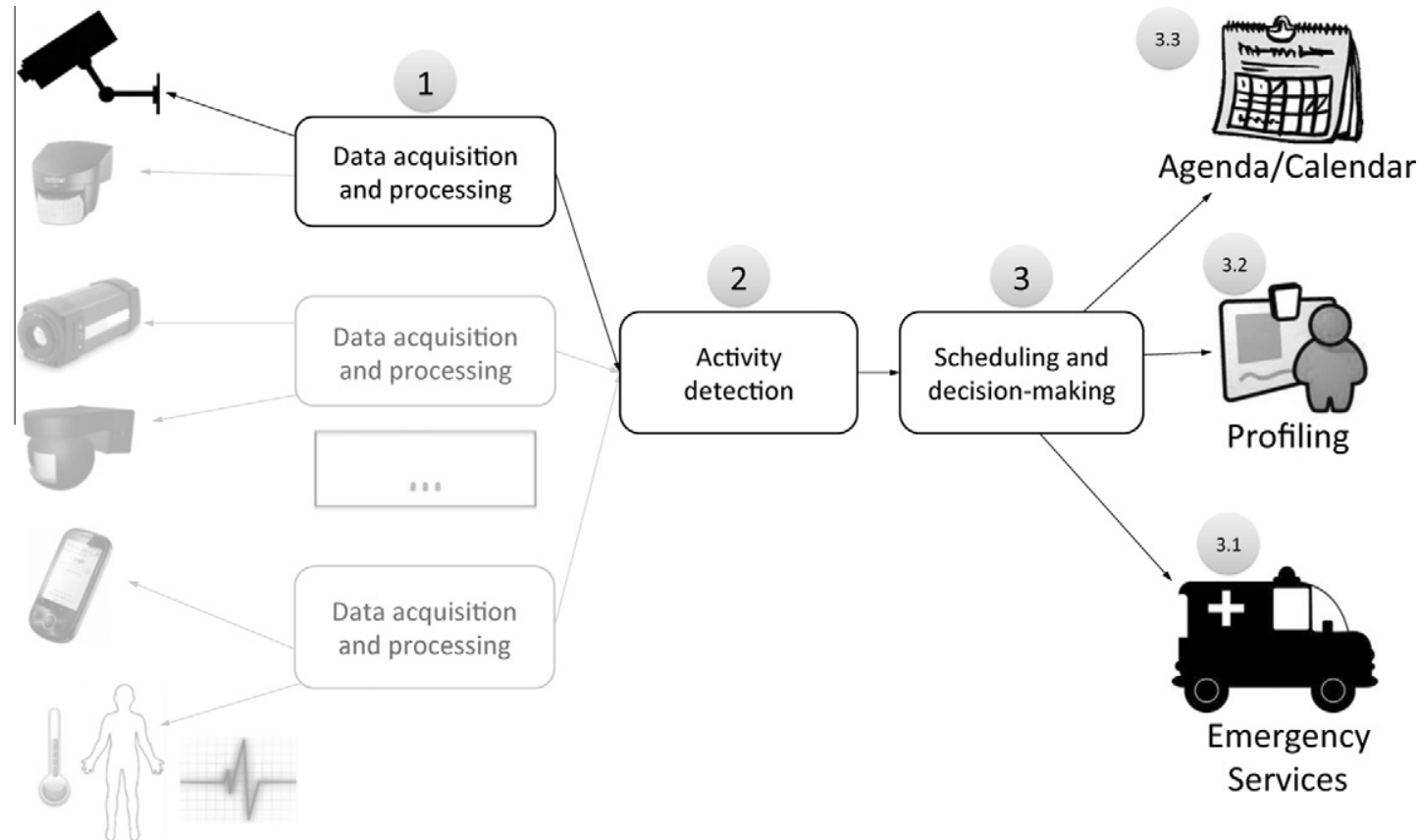

Fig. 8. Case study. Modules and sensors involved.

1. Activate the agents responsible of the warning procedure. The decision system is fully developed in a multi-agent paradigm to maintain flexibility and full proof against errors. The agent receives information containing the importance, place of occurrence and time characteristics.

2. The second step is to enable the questionnaire interface. The interface is projected to be as simple as possible, progressing to a more complete if the user mentions that there is no problem. It leads to three situations:

(a) It is not a fall (false positive) or the fall brought no serious consequences. After the first screen the user is presented a second one to provide more information about the fall, having parameterized fields so the user is as precise as possible. All the information provided is saved in the database. The information in the database is then used to make associations and extract important information and relation about the user and events. (b) The user activates the help in the interface, leading to the event that is saved in the preferences. This can be calling the support center, where trained persons talk with the user using a loudspeaker system to avail the user condition, or directly call the emergency services, providing the user address. Alternatively the system can call a user's relative, if it is configured to do so.

(c) The user does not respond in time. The mobile device is configured to be waiting for a amount of time (configurable); if no interaction is made, the emergency services or support center are notified of the event, so they can proceed accordingly.

A database is developed to save each and every occurrence. This is done with mainly three purposes: updating the user profile, providing useful information about the overall health condition, and providing heuristics for further enhancement of the system.
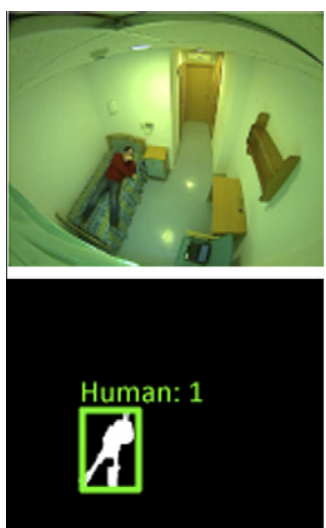

Frame 384
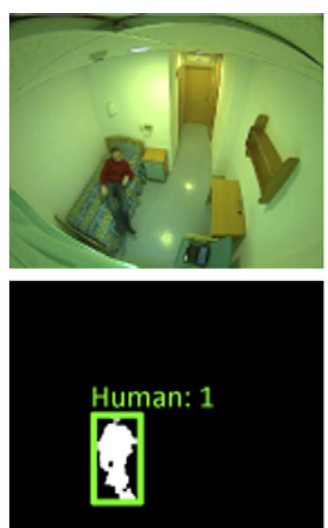

Frame 420
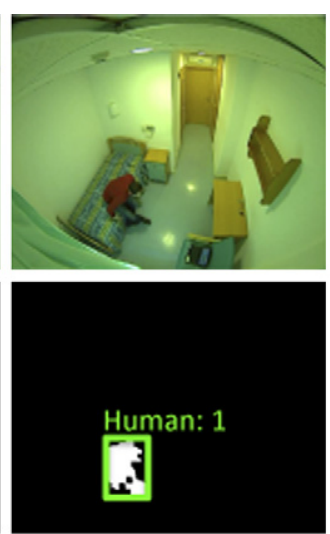

Frame 468
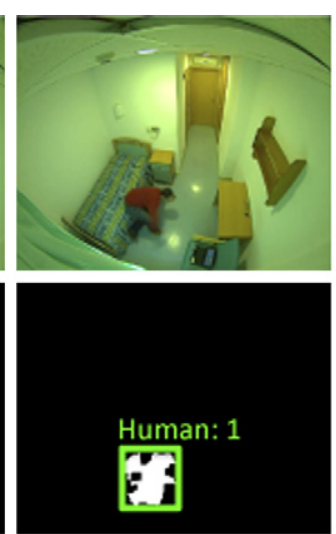

Frame 481

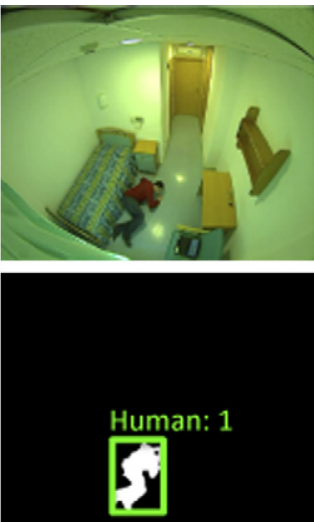

Frame 487

Fig. 9. Human detection. The first row shows the input images. The second row shows the segmentation results along with classification and tracking. 
Table 2

Activity detection.

\begin{tabular}{ll}
\hline \# Frame & Activity \\
\hline 384 & Lying \\
420 & Lying \\
468 & Sitting \\
481 & Crouching \\
487 & Fallen \\
\hline
\end{tabular}

\subsubsection{Profiling system - User profile}

The user profile is developed through $\mathrm{BN}$ and $\mathrm{CBR}$ processing, but to provide accurate decisions and to better respond to the user needs, the information present in the database must be growing and changing when different actions are performed. This is performed in the profiling system component of the platform. The fact is that over time the information changes, because the agents work constantly to optimize the relations of events, being optimized very fast and keeping the same threshold if no new information is sent to the database. Therefore, there are constant additions of the events, actions and activities that the user has done. The principal contributor is the warning mechanism (see Clinical guidelines) by feeding every detected interaction. The second is the agenda manager by perceiving the user interaction with the calendar, directly or indirectly, and saving the preferences of the user towards a scheduled event. If the user chooses to execute the activity or delete or change it, the agenda changes the importance degrees of the event in the database.

Even though the most impacted activities are the playful ones (scheduled by the Free time manager), because they are present in the database, the importance of the person that schedules the activity can also change. All these changes must be in accordance of what the user is doing and his/her interests at that moment. The information present in the database is then a representation of the user full state: personality and health condition. Both aspects are processed by the agenda manager by collecting the information of the database and creating activities and events in the user agenda, subtly orienting the user towards the recommended actions.

\subsubsection{Agenda manager - Appointments}

As presented in the other sections, the Agenda Manager presents a multitude of services oriented to the user wellbeing, by providing intelligent scheduling and a convenient interface, in which the user interacts with the platform. The concept and architecture, abridging the multi-agent paradigm, is highly modular. Thus, other services can connect to this platform and communicate with the present agents.

In line with the architecture of the platform, it is imperative to present the possible outcomes that provide services of interest to the user, whereas his health and health related actions are made. Working with the information present in the database the agenda management entails extracting a lot of information about the user health condition, providing the means to proceed with the convenient actions. Some actions are scheduling an appointment with the user doctor, or report to the relatives a deterioration of the health condition, as depicted in Figs. 11 and 12. Availability of these services is under the possibility of expansion. Albeit they are not numerous, they are quite essential, and, at this point, the interest goes mainly to the procedures of the database parsing and information extraction. It was provided services such as detection of the number of falls and comparing to guidelines that can

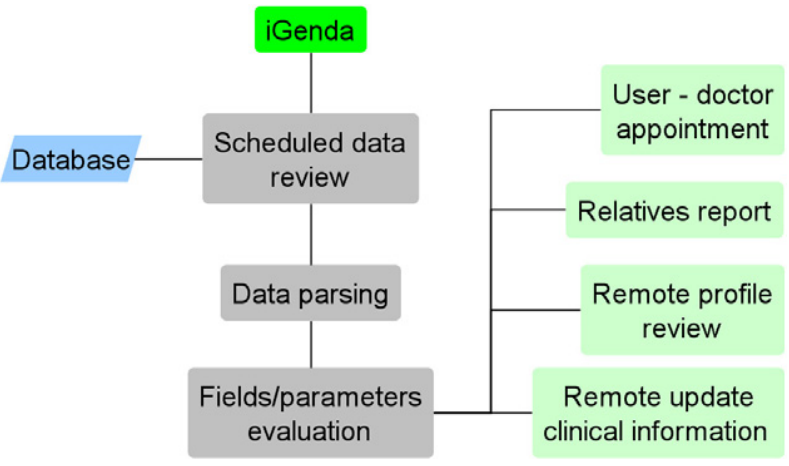

Fig. 11. Structure of the agenda management.

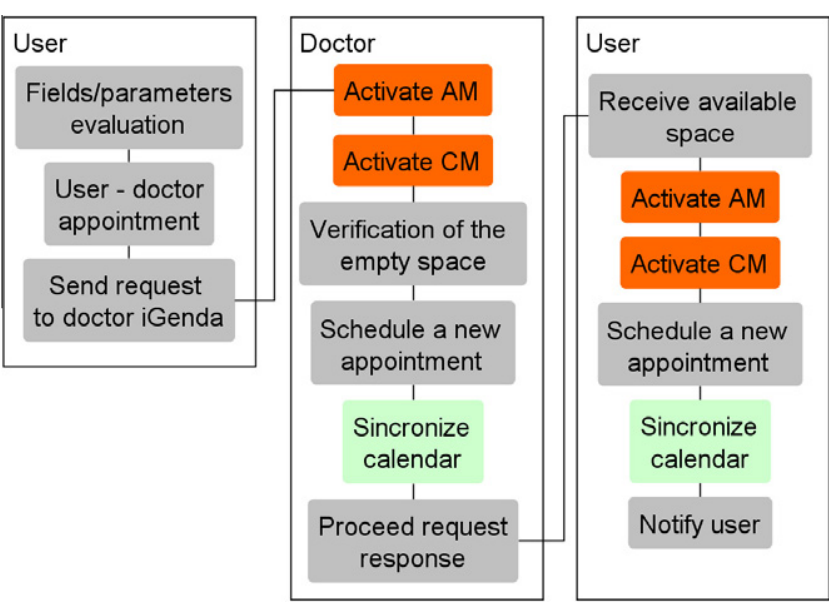

Fig. 12. Structure of automatically scheduling a user-doctor appointment.
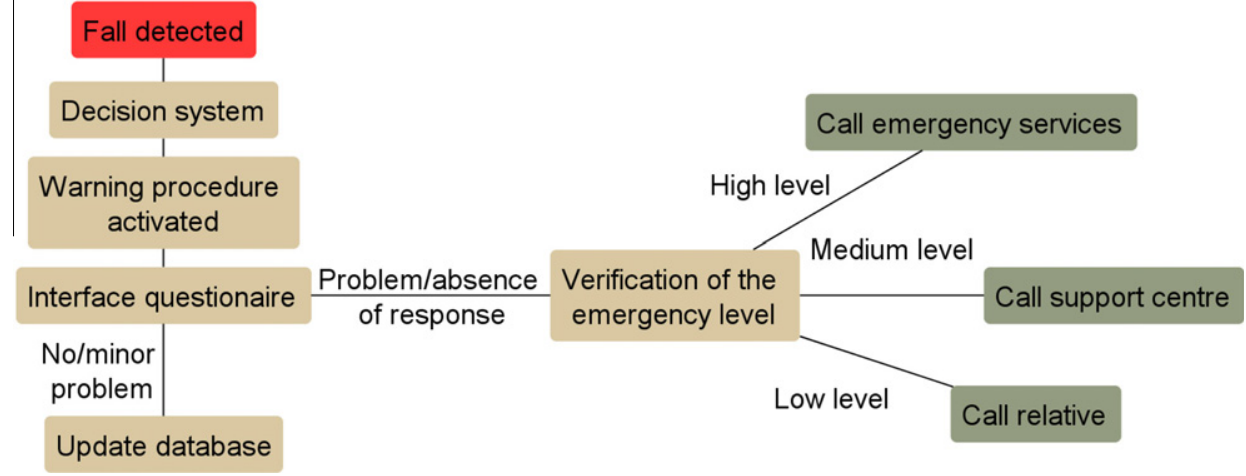

Fig. 10. Structure of the emergency procedure. 
warn a specialized doctor and automatically scheduling an appointment in the doctor and user agenda if there is any problem with the user health condition. These services have quite intricate procedures done by software agents. It requires that the iGenda platform, both the user and the doctor, should be fully functioning and be able to exchange normal events requests.

The method of saving all interactions to a database can be used in the future with different updates to the platform, thus providing new services that help the user in other areas. The platform is developed to be fully functional and solely requires interactions from the user when absolutely required, providing a more independent life.

\section{Conclusions and future work}

This paper has presented an information and communications technology (ICT) solution towards facing the current problem of attending home-bound persons who live alone and are in need of continuous monitoring. In this sense, the concept of Ambient Assisted Living (AAL), which consists of applying technology in a user's home environment, transforming it into a seamless interactive sphere adapted to the user's needs, has inspired the approach offered in this proposal. Indeed, the paper has presented a platform to guide and assist these persons (mostly elderly people) by providing multisensory monitoring and intelligent assistance.

A three-level platform has been described extensively. The lower level ("Data acquisition and processing") performs the usual tasks of a monitoring system, collecting data from the sensors and processing it for the purpose of detecting and tracking humans in order to identify their activities. Initially, a Data acquisition component translates the data captured by the sensors. The Blob detection component filters the spots that are candidates to be objects of interest. The next component is in charge of Object identification, tracking \& classification, raising the information abstraction from blobs to objects. Then, the proposal incorporates external knowledge about the problem domain into the expected structure of an "Activity detection" module. Motion-based image features are explicitly linked to a symbolic notion of hierarchical activity through several layers of more abstract activity descriptions. Here, atomic actions are detected and fed to handcrafted grammars to detect activity patterns of interest. Besides, we work with shape and trajectory to indicate the events related to moving objects. Now, the upper level, "Scheduling and decision-making", consists of a scheduler which provides warnings, intelligently schedules events and serves as an interface to the rest of the platform. The scheduling interface with the user is developed following the principles established in the iGenda project. Intelligent scheduling goes beyond the normal conception of traditional scheduling. The intelligent agenda contains several events in charge of actions that persons normally carry out, such as taking medication, but at the time are difficult to remember for a memory impaired person. Currently, the components of the "Scheduling and decision-making" are Agenda manager, Conflicts manager, Free time manager, profiling system, Clinical guidelines, Medication reminder and Sensing manager.

Moreover, the approach uses mobile and static sensors performing constant monitoring of the user and his/her environment, providing a safe environment and an immediate response to severe problems. The mobile platform is optimally developed to interact with the users, providing visual and audible information, as well as warnings. Also, the mobile platform enables everywhere availability to the user. This means that it is easier to the user to provide feedback to the platform when required, without having to even move from where he/she is. Also, the mobile platform serves as a direct input to an emergency situation. If the user is inactive for a period of time, the platform is prompted to present a question to the user, collecting different aspects beyond the answer itself. The lack of response from the user points out that something happened to the user. Also it can serve as a panic button that calls to a relative or an emergency service, providing automatically the user's location.

Lastly, a case study on elderly fall detection in a nursery home bedroom has shown the usefulness of the proposal. It is our intention to install our platform at different locations of a nursery home and increase the current capacities of the platform. And, although the initial consideration and development was made to face the problems inherent in the elderly, as future work we propose to enhance the functionalities in the mobile platform. We consider working for persons with visual or hearing difficulties. In this sense, we envisage to engage in adaptive interfaces, which is an important approach to build a system that is welcomed by the users, by providing more and more intuitive interfaces.

\section{Acknowledgements}

This work is partially supported by the Spanish Ministerio de Economía y Competitividad/FEDER under Project TIN2010-20845C03-01 and by the Spanish Junta de Comunidades de Castilla-La Mancha/FEDER under Project PII2I09-0069-0994. Also in collaboration with the AAL4ALL QREN 13852 project.

\section{References}

Bajo, J., Fraile, J. A., Pérez-Lancho, B., \& Corchado, J. M. (2010). The THOMAS architecture in home care scenarios: A case study. Expert Systems with Applications, 37(5), 3986-3999.

Beard, J. (2010). A global perspective on population ageing. European Geriatric Medicine, 1(4), 205-206.

Botia, J. A., Villa, A., \& Palma, J. (2012). Ambient assisted living system for in-home monitoring of healthy independent elders. Expert Systems with Applications. http://dx.doi.org/10.1016/j.eswa.2012.01.153.

Castillo, J. C., Serrano-Cuerda, J., \& Fernández-Caballero, A. (2010). Robust people segmentation by static infrared surveillance camera. Lecture Notes in Computer Science, 6096, 348-357.

Castro, J., Delgado, M., Medina, J., \& Ruiz-Lozano, M. (2011). Intelligent surveillance system with integration of heterogeneous information for intrusion detection. Expert Systems with Applications, 38(9), 11182-11192.

Costa, Â., Barbosa, G., Melo, T., \& Novais, P. (2011). Using mobile systems to monitor an ambulatory patient. Advances in Intelligent and Soft Computing, 91, 337-344.

Costa, Â, Castillo, J. C., Novais, P., Fernández-Caballero, A., \& López, María T. (2012). Sensor-Driven intelligent ambient agenda. In P. Novais, K. Hallenborg, D. L. Tapia, \& J. M. C. Rodríguez (Eds.), Ambient Intelligence-Software and Applications (pp. 19-26). Berlin, Heidelberg: Springer.

Costa, Â., Novais, P., Corchado, J. M., \& Neves, J. (2011). Increased performance and better patient attendance in an hospital with the use of smart agendas. Logic Journal of IGPL. http://dx.doi.org/10.1093/jigpal/jzr021.

DeLong, R. P. (2003). Interoperability \& sensor fusion. Naval Engineers Journal, 115(2), 89-104.

Englander, R. (2002). Java and SOAP (1st ed., Vol. 11-12). O'Reilly Media, p. 288.

Fernández-Caballero, A., Castillo, J. C., Martínez-Cantos, J., \& Martínez-Tomás, R. (2010). Optical flow or image subtraction in human detection from infrared camera on mobile robot. Robotics and Autonomous Systems, 58(12), 1273-1281.

Fernández-Caballero, A., Castillo, J. C., \& Rodríguez-Sánchez, J. M. (2010). Human activity monitoring by local and global finite state machines. Expert Systems with Applications, 39(8), 6982-6993.

Fernández-Caballero, A., Gómez, F. J., \& López-López, J. (2008). Road-traffic monitoring by knowledge-driven static and dynamic image analysis. Expert Systems with Applications, 35(3), 701-719.

Foundation for Physical Agents (2002). FIPA ACL Message Representation in XML Specification, Architecture.

Huang, K., Wang, S., Tan, T., \& Maybank, S. (2009). Human behavior analysis based on a new motion descriptor. IEEE Transactions on Circuits and Systems for Video Technology, 19(12), 1830-1840.

Jorge, J. A. (2001). Adaptive tools for the elderly: New devices to cope with ageinduced cognitive disabilities. In Proceedings of the 2001 ECNSF workshop on Universal accessibility of ubiquitous computing providing for the elderly (pp. 6670).

Kolling, A., \& Carpin, S. (2007). Detecting intruders in complex environments with limited range mobile sensors. Lecture Notes in Information and Control, 360, 417-426.

Latta, S. G., Tsunoda, K., Geisner, K., Markovic, R., Bennett, D. A. \& Stone Perez, K. (2010). Gesture Keyboarding. Patent application number 2010019922. 
Leijdekkers, P., \& Gay, V. (2008). A self-test to detect a heart attack using a mobile phone and wearable sensors. In Proceedings of the 21st IEEE international symposium on computer based medical systems (pp. 93-98).

Marques, V., Costa, Â., \& Novais, P. (2011). A dynamic user profiling technique in a AmI environment. In Proceedings of the world congress on information and communication technologies (pp. 1251-1256).

Natarajan, P., \& Nevatia, R. (2008). View and scale invariant action recognition using multiview shape-flow models. In IEEE conference on computer vision and pattern recognition (pp. 1-8).

Nehmer, J., Becker, M., Karshmer, A., \& Lamm, R. (2006). Living assistance systems: An ambient intelligence approach. Proceedings of the 28th international conference on software engineering (pp. 43-50).

Neumann, B., \& Möller, R. (2008). On scene interpretation with description logics. Image and Vision Computing, 26(1), 82-101.

Oliver, N., \& Horvitz, E. (2005). A comparison of HMMs and dynamic Bayesian networks for recognizing office activities. In: Proceedings of the international conference on user modeling (pp. 199-209)

Olivieri, D. N., Gómez Conde, I., \& Vila Sobrino, X. A. (2012). Eigenspace-based fall detection and activity recognition from motion templates and machine learning. Expert Systems with Applications, 39(5), 5935-5945.

Pacciarelli, D., Meloni, C., \& Pranzo, M. (2011). Models and methods for production scheduling in the pharmaceutical industry. International Series in Operations Research \& Management Science, 152, 429-459.
Paoli, R., Fernández-Luque, F. J., Doménech, G., Martínez, F., Zapata, J., \& Ruiz, R. (2012). A system for ubiquitous fall monitoring at home via a wireless sensor network and a wearable mote. Expert Systems with Applications, 39(5), 5566-5575.

Schumacher, J., Salehi, S., Hadjiyanni, T., \& Papanikolopoulos, N. (2011). Rethinking surveillance - toward an interactive security model. In Proceedings of the 19th Mediterranean conference on control \& automation (pp. 688-693).

Ughetti, M., Trucco, T., \& Gotta, D. (2008). Development of agent-based, peer-topeer mobile applications on ANDROID with JADE. In Proceedings of the second international conference on mobile ubiquitous computing systems services and technologies (pp. 287-294).

Ulusoy, I. \& Bishop, C. M. (2005). Generative versus discriminative methods fo object recognition. In Proceedings of the 2005 IEEE conference on computer vision and pattern recognition (Vol. 2, pp. 258-265).

United Nations (2009). World Population Ageing 2009. <http://www.un.org/esa/ population/publications/WPA2009/WPA2009-report.pdf $>$.

Vallejo, D., Albusac, J., Gonzalez-Morcillo, C., \& Jimenez, L. (2008). A service-oriented multiagent architecture for cognitive surveillance. In Lecture Notes in Computer Science (Vol. 5180, pp. 101-115).

Weil, D. N. (2006). Population Aging, National Bureau of Economic Research Working Paper 12147. <http://www.nber.org/papers/w12147.pdf>. 